\title{
Minimal Direct Gauge Mediation
}

November 2007

\author{
Masahiro Ibe ${ }^{1}$ and Ryuichiro Kitano ${ }^{2}$ \\ ${ }^{1}$ Stanford Linear Accelerator Center, Stanford University, Stanford, CA 94309 and \\ Physics Department, Stanford University, Stanford, CA 94305 \\ ${ }^{2}$ Theoretical Division T-8, Los Alamos National Laboratory, Los Alamos, NM 87545
}

\begin{abstract}
We propose a simple model of gauge mediation with a small gravitino mass, $m_{3 / 2}<O(10) \mathrm{eV}$, where supersymmetry is broken by a strong dynamics at $O(10) \mathrm{TeV}$.
\end{abstract}

\section{A. Introduction}

Models with gauge mediated supersymmetry breaking [1, 2, 3, 4] with a very small gravitino mass, $m_{3 / 2}<$ $O(10) \mathrm{GeV}$, are an attractive possibility since it does not cause any cosmological or astrophysical problems [5]. It is, however, difficult to construct a consistent model with such a light gravitino (see Refs. [6, 7, 8, 9, 10, 11, 12, 13. for earlier attempts). In this letter, we propose a simple (and possibly the simplest) model with $m_{3 / 2}<O(10) \mathrm{eV}$, where not only the supersymmetry breaking but also the masses of the messenger particles are generated by the effects of the strong dynamics of a gauge interaction.

\section{B. Dynamical supersymmetry breaking/messenger sector}

A model of the dynamics of the supersymmetry breaking is based on a supersymmetric $\mathrm{SU}(5)_{H}$ gauge theory with five flavors $(F$ and $\bar{F})$ [14], where the subgroup of a global $\mathrm{SU}(5)_{F}$ symmetry $(F: 5$ and $\bar{F}: \overline{5})$ is identified with the gauge groups of the standard model. The only other ingredient of the model is a singlet superfield $S$ which couples to $F$ and $\bar{F}$ in the superpotential,

$$
W=k S F \bar{F},
$$

where $k$ is a coupling constant. $F$ and $\bar{F}$ (or their composite particles) serve as the messenger fields once both the scalar- and the $F$-term of the singlet obtain non-vanishing vacuum expectation values; $\langle S\rangle \neq 0$ and $F_{S} \neq 0$ [28]. Recently, models of the sweet spot supersymmetry [15] based on this dynamical supersymmetry breaking/messenger model have been analyzed in the elementary messenger regime [16] and in the composite messenger regime [17]. In the sweet spot supersymmetry, the supersymmetry breaking local minimum at $\langle S\rangle \neq 0$ is realized by the gravitational stabilization mechanism [18]. In this letter, we seek another possibility of making $\langle S\rangle \neq 0$.

To see how the supersymmetry breaking occurs, let us consider the region where the "messenger mass", $M_{\text {mess }} \equiv k S$, is smaller than the scale $\Lambda$ around which the $\mathrm{SU}(5)_{H}$ gauge interaction becomes strong. In this region, the model can be described by using mesons, $M \sim F \bar{F}$ and baryons $B \sim F^{5}$ and $\bar{B} \sim \bar{F}^{5}$. Here, we omit the indices of the flavor $\mathrm{SU}(5)_{F}$ symmetry. In terms of the mesons and baryons, the full effective superpotential is given by

$$
W_{\mathrm{eff}}=k S \cdot \operatorname{Tr} M+X\left(\operatorname{det} M-B \bar{B}-\left(\Lambda_{\mathrm{dyn}}^{2} / 5\right)^{5}\right),
$$

where $X$ is a Lagrange multiplier field which guarantees the quantum modified constraint between the mesons and baryons [19]. $\Lambda_{\text {dyn }}$ denotes the dynamical scale of $\mathrm{SU}(5)_{H}$ gauge interaction and it is naively related to the scale $\Lambda$ by $\Lambda_{\text {dyn }} \sim \sqrt{N_{c}} \Lambda /(4 \pi)$ with $N_{c}=5$ [20, 21]. By expanding the meson and baryon fields around a solution to the above constraint: $M=\Lambda_{\mathrm{dyn}}\left(\Lambda_{\mathrm{dyn}} \delta_{i j} / 5+\delta \hat{M}\right)$, $(\operatorname{Tr} \delta \hat{M}=0), \hat{B} \sim B / \Lambda_{\text {dyn }}^{4}$ and $\hat{\bar{B}} \sim \bar{B} / \Lambda_{\text {dyn }}^{4}$, we obtain a superpotential,

$$
W_{\text {eff }} \sim k \Lambda_{\text {dyn }}^{2} S+S\left(\frac{k}{2} \operatorname{Tr} \delta \hat{M}^{2}+k \hat{B} \hat{\bar{B}}+\cdots\right),
$$

which has a linear term of the singlet $S$. Here, the ellipses denote the higher dimensional operators of mesons and baryons, and we have neglected non-calculable corrections to the coupling constants which are naively expected to be $O(1)$. This superpotential shows that there is a supersymmetric minimum at $S=0$ and $\delta \hat{M}^{2} \neq 0$ or $\hat{B} \hat{\bar{B}} \neq 0$. However, if the singlet $S$ has a local minimum at $S=\langle S\rangle \gtrsim \Lambda_{\mathrm{dyn}}$, the mesons and baryons have positive masses squared and the spontaneous supersymmetry breaking is achieved by $F_{S} \sim k \Lambda_{\mathrm{dyn}}^{2}$.

Now, the question is: is there a possibility for the singlet $S$ to have a local minimum at $S=\langle S\rangle>\Lambda_{\mathrm{dyn}}$ ? To address this question, notice that there are non-calculable contributions to the effective Kähler potential of the singlet $S$ from the strong interactions below the scale $\Lambda$,

$$
K_{\mathrm{eff}}=S^{\dagger} S+\frac{25 \Lambda^{2}}{(4 \pi)^{2}} \delta K\left(\frac{k S}{\Lambda}\right) .
$$

Here, we have used the "naive dimensional analysis" 20, 21], and we expect that the non-calculable contribution $\delta K(x)$ has no small parameter. From this Kähler potential, we obtain a scalar potential,

$$
V(S) \sim \frac{\left|F_{S}\right|^{2}}{1+25(k / 4 \pi)^{2} \delta K^{(2)}(k S / \Lambda)},
$$

where $\delta K^{(2)}$ denotes the second derivative of $\delta K$ with respect to $k S / \Lambda$. This potential shows that there is a possibility that the potential has a local minimum around $\langle S\rangle \sim \Lambda / k$ which is larger than $\Lambda_{\text {dyn }}$ for $k \lesssim 4 \pi / \sqrt{5}$. 


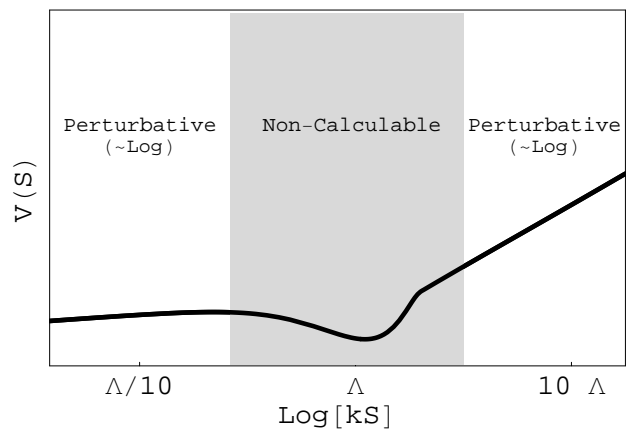

FIG. 1: Schematic picture of the scalar potential $V(S)$. In the dark shaded region, there is a possibility that $V(S)$ has a local minimum due to the non-caluculable contribution to the Kähler potential from the strong dynamics.

Therefore, if we take this possibility positively, it is not hopeless to expect that the singlet $S$ has a local minimum around $\Lambda / k$ by the effect of the non-calculable contributions from the strong dynamics (see Fig. 1).

Note that calculable radiative corrections to the Kähler potential through the diagrams in which the mesons and baryons circulate dominate over the non-caluculable contribution $\delta K$ for a small value of the singlet $S, k S \ll \Lambda$. The potential curves up by this contribution [29]. The masses of the mesons and baryons, however, become comparable to the scale $\Lambda$ around $S \sim \Lambda / k$. Hence, their effects can be overwhelmed by the non-calculable contribution in the region of $S \sim \Lambda / k$. Therefore, the possibility of the local minimum around $S \sim \Lambda / k$ can not be excluded by these effects (see Ref. 23] for a similar discussion).

For $M_{\text {mess }}=k S \gg \Lambda$, the dynamics can be described by using $F$ and $\bar{F}$ as elementary fields. In this region, it can be shown that the potential curves up in the $S$ direction by radiative corrections to the Kahler potential [24]. Therefore, the singlet $S$ cannot have a local minimum at $S \gg \Lambda / k$.

The above discussion does not exclude the possibility of having a local minimum around $S \sim \Lambda / k$ where all the calculable contributions are comparable to the noncalculable ones. Put it all together positively, we here assume that there is a local and supersymmetry breaking minimum around $\langle S\rangle \sim \Lambda / k$, aside from the supersymmetric minimum at $S=0$ [30]. In the following, we consider a model with gauge mediation around the local minimum at

$$
\begin{aligned}
\langle S\rangle & \sim \Lambda / k \sim 4 \pi \Lambda_{\mathrm{dyn}} /(\sqrt{5} k) \gtrsim \Lambda_{\mathrm{dyn}} \\
F_{S} & \sim k \Lambda_{\mathrm{dyn}}^{2} \\
\langle\delta \hat{M}\rangle & =\langle\hat{B}\rangle=\langle\hat{\bar{B}}\rangle=0 .
\end{aligned}
$$

\section{Spectrum of supersymmetric standard model particles}

The mesons $\delta \hat{M}$ transform as the adjoint representation under $\mathrm{SU}(5)_{F}$ whose subgroup is identified with the standard model gauge group, (i.e. $(8,1)_{0},(1,3)_{0}$, $(3,2)_{-5 / 6}$ and $(\overline{3}, \overline{2})_{5 / 6}$ under the standard group). Thus, the mesons mediate the effects of the supersymmetry breaking to the standard model sector via gauge interactions of the standard model. Through loop diagrams of the mesons, we obtain masses of the gauginos and scalar particles in the standard model sector,

$$
\begin{aligned}
m_{\text {gaugino }} & =N_{m} \frac{g^{2}}{(4 \pi)^{2}} \frac{F_{S}}{\langle S\rangle}\left(1+O\left(k^{4}\right)\right), \\
m_{\text {scalar }}^{2} & =2 N_{m} C_{2} \eta\left(\frac{g^{2}}{(4 \pi)^{2}}\right)^{2}\left|\frac{F_{S}}{\langle S\rangle}\right|^{2}\left(1+O\left(k^{4}\right)\right),
\end{aligned}
$$

where $N_{m}=5$ is the sum of the Dynkin index of the mesons, $C_{2}$ is the quadratic Casmir invariant of the scalar particles, and $g$ denotes the gauge coupling constant of the standard model gauge group. As we discussed above, $F_{S} /\langle S\rangle$ is given by $F_{S} /\langle S\rangle \sim \sqrt{5} k^{2} \Lambda_{\text {dyn }} / 4 \pi$. The $O(1)$ coefficient $\eta$ for the scalar masses denotes non-caluculable $O\left(F_{S}^{2} /\langle S\rangle^{2}\right)$ contributions from the heavy hadrons which are charged under the standard model gauge groups, while gaugino masses do not get $O\left(F_{S} /\langle S\rangle\right)$ contributions from them. The $O\left(k^{4}\right)$ contributions come from the diagrams with more $S$ inserted. The gravitino mass, on the other hand, is given by,

$$
m_{3 / 2}=\frac{F_{S}}{\sqrt{3} M_{\mathrm{pl}}} \sim \frac{k \Lambda_{\mathrm{dyn}}^{2}}{\sqrt{3} M_{\mathrm{pl}}},
$$

where $M_{\mathrm{pl}} \simeq 2.4 \times 10^{18} \mathrm{GeV}$ denotes the reduced Planck scale. As a result, we find that the gauge mediation model with $m_{3 / 2}<O(10) \mathrm{eV}$ is achieved by choosing the parameters as,

$$
\begin{aligned}
\Lambda_{\text {dyn }} & \sim 2 \times 10^{5} \mathrm{GeV} \times \eta^{1 / 6}\left(\frac{m_{3 / 2}}{10 \mathrm{eV}}\right)^{2 / 3}\left(\frac{1 \mathrm{TeV}}{m_{\text {squark }}}\right)^{1 / 3}, \\
k & \sim 0.9 \times \eta^{-1 / 3}\left(\frac{10 \mathrm{eV}}{m_{3 / 2}}\right)^{1 / 3}\left(\frac{m_{\text {squark }}}{1 \mathrm{TeV}}\right)^{2 / 3} .
\end{aligned}
$$

Now several comments are in order. The perturbativity of the standard model gauge interactions up to the scale of the Grand Unification Theory (GUT) put a bound on the sum of the Dynkin index of the messenger field $N_{m}$ as

$$
N_{m} \lesssim 150 / \ln \left(M_{\mathrm{GUT}} / M_{\text {mess }}\right),
$$

where $M_{\mathrm{GUT}} \simeq 2 \times 10^{16} \mathrm{GeV}$ denotes the scale of GUT. For $M_{\text {mess }} \sim \Lambda \sim 10^{6} \mathrm{GeV}$, this condition requires $N_{m}$ as $N_{m} \leq 6$. Therefore, the Dynkin index of the present model, $N_{m}=5$, satisfies the perturbative condition of the standard model gauge interactions up to the GUT scale. 
We should also mention the perturbativity of the coupling constant $k$. The coupling constant $k$ becomes small at the high energy scale as a result of the large renormalization effect from the strong gauge interaction of $\mathrm{SU}(5)_{H}$. Thus, we can expect the coupling constant $k$ stays perturbative up to around the GUT scale, although it is not necessarily required [31].

The tunneling rate to the supersymmetric vacuum at $S=0$ per unit volume is roughly given by $\Gamma / V \sim\langle S\rangle^{4} e^{-\mathcal{S}_{E}}$, where $\mathcal{S}_{E}$ is estimated by $\mathcal{S}_{E} \sim$ $2 \pi^{2}\langle S\rangle^{4} / V(S) \sim 2 \pi^{2}(4 \pi)^{4} /\left(5^{2} k^{6}\right) \sim 10^{5}$ for $k \sim 0.9$. On the other hand, the vacuum stability condition within the observable volume and over the age of the universe only requires $\mathcal{S}_{E} \gtrsim 400$. Thus, although our vacuum is not stable quantum mechanically, it has a lifetime much longer than the age of the universe.

Finally, we comment on the effects of the supergravity to the scalar potential of $S$. The leading effect of the supergravity comes from the linear term of the singlet $S$ in the superpotential which leads to a linear term in the scalar potential,

$$
V(S)_{\text {linear }}=2 m_{3 / 2} k \Lambda_{\mathrm{dyn}}^{2} S+\text { h.c. }
$$

The linear term, however, is negligible compared with the scalar potential in Eq. (5) around $S \sim \Lambda / k$ as long as,

$$
k \gtrsim 4\left(\frac{\Lambda_{\mathrm{dyn}}}{M_{\mathrm{pl}}}\right)^{1 / 3} .
$$

Here we have used $\partial V(S) / \partial S \sim 5(\sqrt{5} k / 4 \pi)^{3}\left|F_{S}\right|^{2} / \Lambda_{\text {dyn }}$ for $S \sim 4 \pi \Lambda_{\mathrm{dyn}} /(\sqrt{5} k)$. The condition is easily satisfied for $m_{3 / 2}=O(10) \mathrm{eV}$ (Eq. (12)), and hence, the local minimum we chose is stable against the supergravity effects.

On the other hand, the linear term plays an important role to generate the mass of the $R$-axion which corresponds to the spontaneous breaking of the $R$-symmetry by $S \neq 0$. Since the $R$-symmetry is broken explicitly by the linear term, the $R$-axion obtains a mass [25],

$$
\begin{aligned}
m_{a} & \simeq 2 m_{3 / 2}\left(\frac{M_{\mathrm{pl}}}{\langle S\rangle}\right)^{1 / 2} \\
& \sim 40 \mathrm{MeV}\left(\frac{m_{3 / 2}}{10 \mathrm{eV}}\right)^{5 / 6}\left(\frac{m_{\text {squark }}}{1 \mathrm{TeV}}\right)^{1 / 2} \eta^{-1 / 4}
\end{aligned}
$$

The $R$-axion couples to the standard model particles through the loop diagrams of mesons which are relevant for the gauge mediation. As a result, it decays mainly into photons at the cosmic temperature $T \sim O(10) \mathrm{MeV}$. Note that for the axion with mass $m_{a} \sim O(10) \mathrm{MeV}$, final states with hadrons or electroweak gauge bosons are kinematically forbidden. The cosmic abundance of the $R$-axion before the decay (both from thermal and nonthermal production) is estimated to be small enough that the decay does not cause a large entropy production 32]. The decay temperature is also high enough not to spoil the success of the Big-Bang Nucleosynthesis. Besides, the above $R$-axion marginally satisfies an astrophysical constraint based on stellar cooling rate and supernova dynamics: $m_{a} \gtrsim O(10) \mathrm{MeV}$ [26]. Therefore, we find that the $R$-axion in our model does not cause any cosmological and astrophysical problems.

\section{Conclusions}

We find a very simple model with a very small gravitino mass $\left(m_{3 / 2}<O(10) \mathrm{eV}\right)$, where both the supersymmetry breaking and the mass of the messenger particles are generated by the strong dynamics. A light gravitino in this range are very attractive for cosmology and astrophysics. Furthermore, such a small gravitino mass can be determined at the future collider experiments such as LHC/ILC, by measuring the branching ratio of the decay rate of the next to lightest superparticle [27].

Finally, it should be noted that the present model is also applicable for a wide range of the gravitino mass up to $m_{3 / 2} \sim O(10) \mathrm{MeV}$, where the condition in Eq. (15) is saturated (i.e. $k \sim 10^{-2}, \Lambda_{\text {dyn }} \sim 10^{10} \mathrm{GeV}$ ).

\section{Acknowledgments}

MI thanks Y. Nakayama and T.T. Yanagida for useful discussion. The work of MI was supported by the U.S. Department of Energy under contract number DE-AC0276 SF00515.
[1] M. Dine, W. Fischler and M. Srednicki, Nucl. Phys. B 189, 575 (1981); S. Dimopoulos and S. Raby, Nucl. Phys. B 192, 353 (1981); M. Dine and W. Fischler, Phys. Lett. B 110, 227 (1982); Nucl. Phys. B 204, 346 (1982); C. R. Nappi and B. A. Ovrut, Phys. Lett. B 113, 175 (1982); L. Alvarez-Gaume, M. Claudson and M. B. Wise, Nucl. Phys. B 207, 96 (1982); S. Dimopoulos and S. Raby, Nucl. Phys. B 219, 479 (1983).

[2] M. Dine and A. E. Nelson, Phys. Rev. D 48, 1277 (1993) arXiv:hep-ph/9303230.

[3] M. Dine, A. E. Nelson and Y. Shirman, Phys. Rev. D 51,
1362 (1995) arXiv:hep-ph/9408384.

[4] M. Dine, A. E. Nelson, Y. Nir and Y. Shirman, Phys. Rev. D 53, 2658 (1996) arXiv:hep-ph/9507378.

[5] M. Viel, J. Lesgourgues, M. G. Haehnelt, S. Matarrese and A. Riotto, Phys. Rev. D 71, 063534 (2005) arXiv:astro-ph/0501562.

[6] K. I. Izawa, Prog. Theor. Phys. 98, 443 (1997) hep-ph/9704382.

[7] K. I. Izawa, Y. Nomura, K. Tobe and T. Yanagida, Phys. Rev. D 56, 2886 (1997) hep-ph/9705228.

[8] Y. Nomura and K. Tobe, Phys. Rev. D 58, 055002 (1998) 
hep-ph/9708377.

[9] K. I. Izawa, Y. Nomura and T. Yanagida, Phys. Lett. B 452, 274 (1999) hep-ph/9901345.

[10] K. I. Izawa and T. Yanagida, Prog. Theor. Phys. 114, 433 (2005) arXiv:hep-ph/0501254.

[11] H. Murayama and Y. Nomura, Phys. Rev. Lett. 98, 151803 (2007) arXiv:hep-ph/0612186; H. Murayama and Y. Nomura, Phys. Rev. D 75, 095011 (2007) arXiv:hep-ph/0701231.

[12] M. Ibe, Y. Nakayama and T. T. Yanagida, Phys. Lett. B 649, 292 (2007) arXiv:hep-ph/0703110.

[13] Y. Nakayama, M. Taki, T. Watari and T. T. Yanagida, Phys. Lett. B 655, 58 (2007) arXiv:0705.0865 [hep-ph]].

[14] K. I. Izawa and T. Yanagida, Prog. Theor. Phys. 94, 1105 (1995) arXiv:hep-ph/9507441.

[15] M. Ibe and R. Kitano, JHEP 0708, 016 (2007) arXiv:0705.3686 [hep-ph]].

[16] Y. Nomura and M. Papucci, arXiv:0709.4060 [hep-ph].

[17] M. Ibe and R. Kitano, arXiv:0710.3796 [hep-ph].

[18] R. Kitano, Phys. Lett. B 641, 203 (2006) arXiv:hep-ph/0607090.

[19] K. A. Intriligator and P. Pouliot, Phys. Lett. B 353, 471 (1995) arXiv:hep-th/9505006.

[20] M. A. Luty, Phys. Rev. D 57, 1531 (1998) arXiv:hep-ph/9706235.

[21] A. G. Cohen, D. B. Kaplan and A. E. Nelson, Phys. Lett. B 412, 301 (1997) arXiv:hep-ph/9706275.

[22] D. Shih, arXiv:hep-th/0703196.

[23] Z. Chacko, M. A. Luty and E. Ponton, JHEP 9812, 016
(1998) arXiv:hep-th/9810253.

[24] N. Arkani-Hamed and H. Murayama, Phys. Rev. D 57, 6638 (1998) arXiv:hep-th/9705189.

[25] J. Bagger, E. Poppitz and L. Randall, Nucl. Phys. B 426, 3 (1994) arXiv:hep-ph/9405345.

[26] G. G. Raffelt, Phys. Rept. 198, 1 (1990).

[27] K. Hamaguchi, S. Shirai and T. T. Yanagida, Phys. Lett. B 651, 44 (2007) arXiv:0705.0219 [hep-ph]].

[28] We use the same notation for chiral superfields and their scalar parts interchangeably.

[29] To ensure the origin of the singlet is not a local minimum in models with $R$-symmetry like our model, we need to introduce fields with $R$-charges other than 0 or 2 [22], although we do not require the instability of the origin of the singlet in our model.

[30] Here, we are also assuming that the Kähler metrics of $\delta \hat{M}, \hat{B}$ and $\hat{\bar{B}}$ are positive definite around the local minimum, although the composite description is not quite well for $k\langle S\rangle \sim \Lambda$.

[31] We cannot trace the renormalization group evolution from the value in Eq. (12) since there is non-calculable $O(1)$ threshold corrections between the value of $k$ at the scale $\Lambda$ and the one at the scale higher than $\Lambda$.

[32] The decay mode into gravitinos are suppressed by a helicity suppression factor of $\left(m_{3 / 2} / m_{a}\right)^{2}$, and hence, the gravitino abundance produced by the decay of the $R$ axion is negligible compared with the one from the thermal bath [25]. 